\title{
On the Validity of Cosmological Fisher Matrix Forecasts
}

\section{Laura Wolz,, 1,2,3 Martin Kilbinger, ${ }^{4,2,3}$ Jochen Weller ${ }^{2,3,5}$ and Tommaso Giannantonio ${ }^{2,3}$}

\author{
${ }^{1}$ Department of Physics and Astronomy, University College London, London WC1E 6BT, \\ UK \\ ${ }^{2}$ Universitäts-Sternwarte, Ludwig-Maximilians-Universität München, Scheinerstr. 1, 81679 \\ München, Germany \\ ${ }^{3}$ Excellence Cluster Universe, Boltzmannstr. 2, 85748 Garching, Germany \\ ${ }^{4}$ CEA Saclay, Service d'Astrophysique (SAp), Orme des Merisiers, Bât. 709, 91191 Gif-sur- \\ Yvette, France \\ ${ }^{5}$ Max-Planck-Institut für extraterrestrische Physik, Giessenbachstr., 85748 Garching, Ger- \\ many \\ E-mail: lwolz@star.ucl.ac.uk, martin.kilbinger@cea.fr, jochen.weller@usm.lmu.de, \\ tommaso@usm.lmu.de
}

\begin{abstract}
We present a comparison of Fisher matrix forecasts for cosmological probes with Monte Carlo Markov Chain (MCMC) posterior likelihood estimation methods. We analyse the performance of future Dark Energy Task Force (DETF) stage-III and stageIV dark-energy surveys using supernovae, baryon acoustic oscillations and weak lensing as probes. We concentrate in particular on the dark-energy equation of state parameters $w_{0}$ and $w_{a}$. For purely geometrical probes, and especially when marginalising over $w_{a}$, we find considerable disagreement between the two methods, since in this case the Fisher matrix can not reproduce the highly non-elliptical shape of the likelihood function. More quantitatively, the Fisher method underestimates the marginalized errors for purely geometrical probes between 30\%-70\%. For cases including structure formation such as weak lensing, we find that the posterior probability contours from the Fisher matrix estimation are in good agreement with the MCMC contours and the forecasted errors only changing on the $5 \%$ level. We then explore non-linear transformations resulting in physically-motivated parameters and investigate whether these parameterisations exhibit a Gaussian behaviour. We conclude that for the purely geometrical probes and, more generally, in cases where it is not known whether the likelihood is close to Gaussian, the Fisher matrix is not the appropriate tool to produce reliable forecasts.
\end{abstract}

Keywords: Dark energy experiments, Supernovae Type Ia, Baryon acoustic oscillations, Gravitational lensing, Cosmological parameters from LSS 


\section{Introduction}

Forecasting cosmological observations has in recent years become an important contribution to the design of future surveys, in particular with respect to revealing the nature of the observed cosmic acceleration. Within this context, the Dark Energy Task Force [1, hereafter DETF] compared various stages of present and future surveys and cosmological probes. They proposed a figure of merit (FoM), which allows one to compare these surveys in their ability to constrain the equation of state of dark energy in the so-called Chevallier-Polarski-Linder (CPL) parameterisation $[2,3]$.

In most cases, such forecast exercises exploit a second-order approximation to the likelihood, where the parameter covariance is given by the Fisher information matrix [4-6]. This is usually the method of choice, because it is fast and easy to implement, and the results of which are straightforwardly reproduced. However, since the Fisher matrix is a local approximation of the likelihood, its application can lead to a spurious breaking of some - even infinite - degeneracies. This has been long known, for example in the case of cosmic microwave background (CMB) anisotropies. If one neglects the integrated Sachs-Wolfe (ISW) effect on large scales, the CMB can not simultaneously constrain the dark-energy parameters and the curvature of the Universe. However, a Fisher matrix approach would give relatively tight constraints on quantities which in reality are infinitely degenerate.

The DETF provides a prescription to circumvent this problem: instead of using the parameters of dark energy and curvature directly, they propose to take as a variable the angular size of the sound horizon at last scattering, which is well constrained, and whose associated likelihood distribution is approximately Gaussian. The parameter space is then expanded with a suitably chosen Jacobian to its full dimensionality. This by construction leads to the desired, infinite degeneracies $[1,7]$. Interestingly, the angular size of the sound horizon is also a 'good' variable to explore the posterior likelihood efficiently with Monte Carlo methods $[8,9]$.

Prominent cosmological probes other than the CMB are the large-scale distribution of galaxies and the baryon acoustic oscillation (BAO) feature in the power spectrum [10], weak cosmological lensing (WL) or cosmic shear [11, 12], the spatial and redshift distribution of galaxy clusters [13] and the magnitude-redshift relation as measured with Type Ia Supernovae (SN) [14-16]. All these probes measure cosmological parameters with either geometric distance measures, the growth of structures, or a mixture thereof [17]. However, for these probes the Gaussian nature of the likelihood functions is less explored than in the case of CMB anisotropies. Recently, Ref. [18] presented a study in the context of cosmic shear forecasts, which allows to treat mildly non-Gaussian distributions by non-linear parameter transformations.

A more robust approach to the forecast problem is possible, by sampling the full likelihood function without approximations, as was recently used e.g. by Refs. [19-21]. In order to assess the question of how valid a standard Fisher matrix approach is in forecasting constraints, we investigate a selection of future probes, comparing for each case a direct likelihood exploration using a Monte Carlo Markov Chain (MCMC) approach with the Fisher matrix approximation. We will also describe possible alternative parameterisations in which the likelihood takes an approximate Gaussian shape and hence becomes more suitable to a Fisher matrix approach.

This study will be of importance for forecasts for future probes. In the coming decade, 
many new and ever larger surveys are planned and carried out, like Pan-STARRS ${ }^{1}, \mathrm{KiDS}^{2}$,

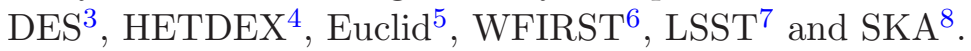

The paper is organised as follows. In Section 2 we briefly describe the methods to explore the likelihood with a MCMC method and the Fisher matrix approximation. Section 3 presents the cosmological probes and surveys we investigate. In Section 4 we sketch a possible alternative parameterisation to obtain a more Gaussian likelihood, followed by a discussion and our conclusions in Section 5.

\section{Forecasting: Fisher matrix vs. Monte Carlo sampling}

To investigate the distribution of a set of parameters $\boldsymbol{\theta}$ under the assumption of a certain (cosmological) model $M$ and given the data $\mathbf{D}$, we have to estimate the posterior probability $\mathcal{P}(\boldsymbol{\theta}) \equiv p(\boldsymbol{\theta} \mid \mathbf{D}, M)$. This is given by Bayes' theorem as a function of the likelihood $\mathcal{L}(\boldsymbol{\theta}) \equiv$ $p(\mathbf{D} \mid \boldsymbol{\theta}, M)$, the prior $\Pi(\boldsymbol{\theta}) \equiv p(\boldsymbol{\theta} \mid M)$ and the Bayesian evidence $\mathcal{Z} \equiv p(\mathbf{D} \mid M)$, as

$$
\mathcal{P}(\boldsymbol{\theta})=\frac{\mathcal{L}(\boldsymbol{\theta}) \Pi(\boldsymbol{\theta})}{\mathcal{Z}} .
$$

If we are interested in parameter estimation for a fixed model only, then the evidence $\mathcal{Z}$ is a trivial normalising factor and can be neglected. The priors are likewise irrelevant for our discussion, and we will take them to be flat in the chosen parameterisation. The likelihood function can be sampled in principle by scanning the entire parameter space on a grid and calculating its corresponding values. However, this is very inefficient and for high-dimensional parameter spaces not feasible. An efficient method to circumvent this problem is the Monte Carlo sampling of the likelihood with the help of a Markov chain.

\subsection{MCMC implementation}

We implement a Monte Carlo Markov Chain by using the Metropolis-Hastings algorithm $[22,23]$. Using a Monte-Carlo integration, the expectation of any function $f(\boldsymbol{\theta})$ can be estimated as

$$
\int f(\boldsymbol{\theta}) \mathcal{P}(\boldsymbol{\theta}) \mathrm{d}^{n} \theta \simeq \frac{1}{N} \sum_{i=1}^{N} f\left(\boldsymbol{\theta}^{(i)}\right),
$$

where the Markov Chain points $\left\{\boldsymbol{\theta}^{(i)}\right\}_{i=1}^{N}$ represent a sample under the posterior $\mathcal{P}(\boldsymbol{\theta})$.

Given the point in the chain $\boldsymbol{\theta}^{(i)}$, a new point $\boldsymbol{\theta}^{\star}$ is drawn from a distribution $\psi\left(\boldsymbol{\theta}^{(i)}-\boldsymbol{\theta}^{\star}\right)$. The new point is accepted as $\boldsymbol{\theta}^{(i+1)}$ with the probability $\min \left\{1, \mathcal{P}\left(\boldsymbol{\theta}^{\star}\right) / \mathcal{P}\left(\boldsymbol{\theta}^{(i)}\right)\right\}$. We choose as the so-called proposal density $\psi$ a multi-variate Gaussian with a diagonal covariance. As a criterion for the convergence of the chains we implement the Gelman-Rubin test [24] by running five independent chains with over-dispersed seeds. Calculating $R$, which is proportional to the ratio of the parameter variances of one chain to the mean parameter variances

\footnotetext{
${ }^{1}$ http://pan-starrs.ifa.hawaii.edu/public

${ }^{2}$ http: //www . astro-wise.org/projects/KIDS

${ }^{3}$ www . darkenergysurvey . org

${ }^{4}$ http: //hetdex.org

${ }^{5}$ www.euclid-ec.org

${ }^{6}$ http://wfirst.gsfc.nasa.gov

${ }^{7}$ http://www. lsst.org/lsst

${ }^{8}$ http://www.skatelescope.org
} 
of the independent chains, yields a quick check of convergence. We consider a chain to be converged when $\sqrt{R}-1<0.05$.

We run MCM chains for each of the cosmological probes: supernovae, BAO and weak lensing, as described in Section 3. The chains are then analysed by investigating the twodimensional joint marginalised posterior distributions, and compared to the Fisher matrix results.

\subsection{Fisher matrix formalism}

For a posterior distribution $\mathcal{P}(\boldsymbol{\theta})$ the Fisher information matrix $[4,5]$ is defined as the expectation value

$$
F_{\alpha \beta}=\left\langle\frac{\partial^{2}[-\ln \mathcal{P}(\boldsymbol{\theta})]}{\partial \theta_{\alpha} \partial \theta_{\beta}}\right\rangle .
$$

We use wide uniform priors on the parameters throughout, therefore the notions of posterior and likelihood are interchangeable.

The inverse of the Fisher matrix is the curvature of the likelihood evaluated at the mean. Usually, the mean is assumed to be equivalent to the maximum likelihood (ML) estimator. $F^{-1}$ is thus a local measure of how fast the likelihood falls off from the maximum in different directions. According to the Cramér-Rao inequality, the Fisher matrix gives an upper bound on the parameter error $\Delta$ on a parameter $\theta_{\alpha}$,

$$
\Delta \theta_{\alpha} \leq \sqrt{\left(F^{-1}\right)_{\alpha \alpha}} .
$$

These two properties make the Fisher matrix a first-order, optimistic approximation of the likelihood. It is calculated very quickly, in particular compared to estimating or sampling the full posterior distribution, if the dimensionality of the parameter space is high. Therefore, the Fisher matrix is used in most cases of parameter forecasts, such as e.g. for predictions of the performance of planned surveys, which play a crucial role in defining the science case and informing funding agencies.

By construction, the Fisher matrix is expected to give, more or less, inaccurate results for non-Gaussian posteriors. In this case, the predicted errors will most likely be too small, and parameter degeneracies could be misestimated, since they are only considered to be linear. This might lead to regions in parameter space to be spuriously excluded, where in fact they are permitted by the full posterior, and vice-versa.

\section{Cosmological Probes}

Some of the major cosmological probes which in recent years have tested scenarios of cosmic acceleration are the observation of the cosmological shearing of galaxy shapes [25], the largescale distribution of galaxies and the associated baryon acoustic feature [26-28], and the magnitude-redshift diagram of SN of type Ia [16, 29-31]. In this work, we model forecasts of these three probes for future surveys. The following section briefly describes the surveys. Details of the settings can be found in Table 1.

Our cosmological model is a flat $w \mathrm{CDM}$ model with a dark-energy equation of state evolving linearly with the scale factor $a, w(a)=w_{0}+w_{a}(1-a)[2,3]$. We vary the total matter density $\Omega_{\mathrm{m}}$ and the two dark-energy parameters, using the fiducial model $\left\{\Omega_{\mathrm{m}}, w_{0}, w_{a}\right\}=$ $\{0.3,-1,0\}$. For weak lensing, we include $\sigma_{8}$ in the analysis, with fiducial value $\sigma_{8}=0.8$. We keep fixed the baryon density $\Omega_{\mathrm{b}}=0.0462$, the Hubble parameter $h=0.72$ and the 


\begin{tabular}{|c|c|c|c|c|c|c|c|c|}
\hline Stage & Experiment & $\Omega_{\text {sky }}\left[\mathrm{deg}^{2}\right]$ & $z_{\min }$ & $z_{\max }$ & $z$ bins & $\left\langle|\gamma|^{2}\right\rangle^{1 / 2}$ & gal. dens. & $z_{\text {med }}$ \\
\hline III & DES & 5,000 & 0.01 & 2.0 & 5 & 0.16 & 12 arcmin $^{-2}$ & 0.8 \\
III & HETDEX & 420 & 1.8 & 3.9 & 10 & - & - & - \\
IV & Euclid (photo) & 15,000 & 0.01 & 2.5 & 8 & 0.247 & $30 \operatorname{arcmin}^{-2}$ & 0.8 \\
IV & SKA & 20,000 & 0.01 & 1.5 & 10 & - & - & - \\
\hline
\end{tabular}

Table 1. Survey settings used for producing the forecasts.

spectral index of primordial density perturbations $n_{\mathrm{s}}=0.96$, corresponding to the WMAP7 values [32].

\subsection{The surveys}

The Dark Energy Survey (DES) [33] will observe 5,000 square degrees in the Southern sky, starting in late 2012. This DETF stage-III imaging survey consists of the five optical bands $g, r, i, z$ and $Y$, using the newly-built DECam on the $4 \mathrm{~m}$ Blanco telescope at the Cerro Tololo Inter-American Observatory (CTIO). The four main cosmological probes of DES are weak gravitational lensing, baryon acoustic oscillations, clusters of galaxies and Type Ia supernovae.

The Hobby-Eberly Telescope Dark Energy Experiment (HETDEX) [34] is a deep optical spectroscopic survey designed to measure the expansion rate, the angular diameter distance and the growth of structure in the redshift range $2<z<4$ to high accuracy. It will observe 420 square degrees on the Northern hemisphere and measure the redshift of 0.8 million $\mathrm{Ly}_{\alpha^{-}}$ emitting galaxies. HETDEX is a DETF stage-III survey, expected to be completed by the end of 2014.

Euclid [35] is a satellite with a $1.2 \mathrm{~m}$ mirror, scheduled as medium-class mission by the European Space Agency (ESA) for launch in 2019. This stage-IV survey will observe 15,000 square degrees of extra-galactic sky with optical and near-infrared imaging, and near-infrared spectroscopy. We will consider here the photometric part only; the imaging filters consist of one broad optical band $(R+I+Z)$ and the three infrared filters $Y, J$ and $H$. To obtain photometric redshifts, Euclid will be complemented by ground-based optical surveys such as DES or Pan-STARRS. The two main science drivers for Euclid are weak lensing and galaxy clustering (BAO and redshift-space distortions) as cosmological probes.

The planned radio interferometer Square Kilometre Array (SKA) [36, 37] is a stage-IV survey designed to measure the 21-centimeter spectral line of neutral hydrogen (HI). SKA's key features are to access the epoch of reionisation and to probe the large-scale structure of HI-emitting galaxies on a sky area of 20,000 square degrees.

\subsection{Supernovae}

Supernovae of type Ia are explosions of white dwarf stars at or near the Chandrasekhar mass limit of $\sim 1.4 M_{\odot}$. The uniform progenitor mass makes them standardisable candles, and they can therefore be used as geometrical measures for cosmological models after rescaling for the stretch of their light curves [38]. In this work, a supernova survey with 1,000 objects equally spaced in the redshift range $0<z<1.8$ is taken as reference; this scenario corresponds to a DETF stage-IV forecast. The observed magnitudes are modeled, ignoring for our purposes the effects of reddening and higher-order stretch corrections, as [14]

$$
m(z)=M_{\text {int }}+5 \log _{10}\left[H_{0} d_{\mathrm{L}}(z)\right] ;
$$



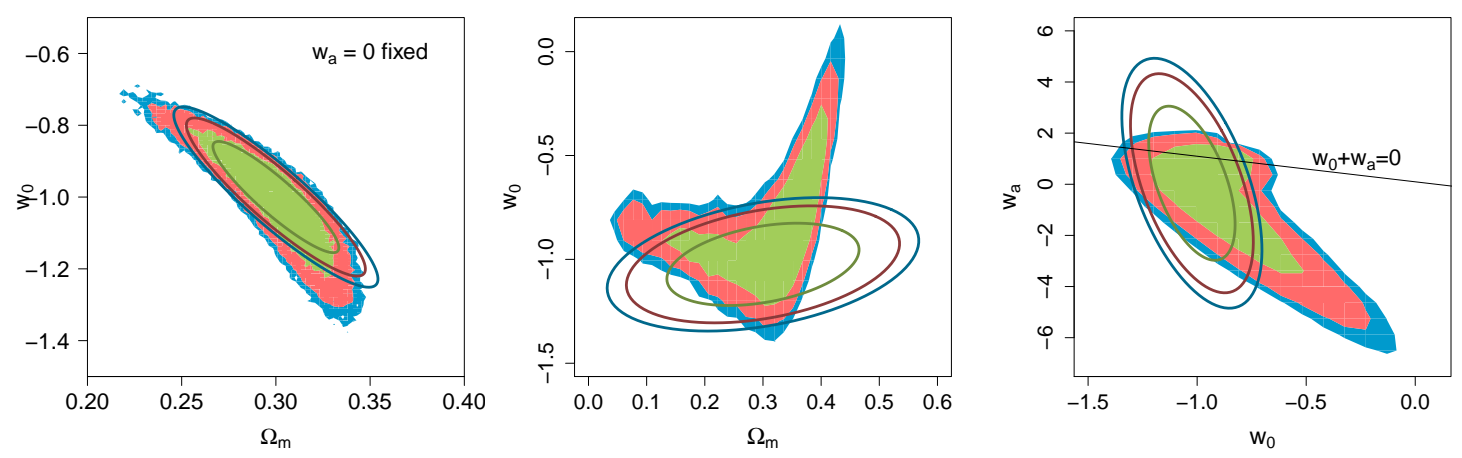

Figure 1. 68\%, 90\% and 95\% confidence regions for a supernova survey. Filled contours correspond to the full posterior sampled with MCMC, while the solid lines represent the Fisher matrix results. The parameter spaces are $\left\{\Omega_{\mathrm{m}}, w_{0}, M_{\mathrm{int}}\right\}$ with fixed $w_{a}=0$ (left panel), and $\left\{\Omega_{\mathrm{m}}, w_{0}, w_{a}, M_{\mathrm{int}}\right\}$ (middle and right panels). The parameters which are not shown have been marginalised in all panels.

here $M_{\mathrm{int}}$ is the intrinsic magnitude of the supernovae and the luminosity distance is

$$
d_{L}(z)=c(1+z) \int_{0}^{z} \frac{d z^{\prime}}{H\left(z^{\prime}\right)}
$$

where the Hubble expansion rate is given for our parameterised dark-energy model by:

$$
\frac{H(z)}{H_{0}}=\sqrt{\Omega_{\mathrm{m}}(1+z)^{3}+\Omega_{\Lambda} e^{-3 w_{a} \frac{z}{1+z}}(1+z)^{3\left(1+w_{0}+w_{a}\right)}} .
$$

Note that we fix $H_{0}$ for the likelihood analysis because it is completely degenerate with the intrinsic magnitude.

To calculate the likelihood, we independently draw 1,000 supernova magnitudes from a Gaussian distribution with variance $\sigma_{m}=0.15$. We consider the case of a fixed $w_{a}=0$ for the likelihood analysis, and the case where $w_{a}$ is fit jointly with the other cosmological parameters. Here we marginalise over the modified intrinsic magnitude $\mathcal{M}=M_{\text {int }}-5 \log H_{0}$. The results are displayed in Fig. 1, showing two-dimensional marginals from MCMC compared to the Fisher matrix ellipses. In the left panel, where we fix $w_{a}=$ const $=0$, the Fisher matrix result for the $\Omega_{\mathrm{m}}-w_{0}$ joint likelihood is a fair approximation of the full likelihood. However, it is substantially smaller, in particular at the $>90 \%$ level. This is in strong contrast to the second case shown in the middle panel: when marginalising over $w_{a}$, the Fisher prediction is completely misleading. The curvature of the likelihood function is highly variable over the parameter space, and the corresponding bends in the contours cause very different parameter regions to be covered compared to the Fisher ellipsoid. We will explain the parameter degeneracies in more detail in Section 3.3, describing the similar behaviour for the BAO forecasts. The right panel of Fig. 1 shows the comparison of the techniques in the $w_{0}-w_{a}$ space. Although the total sizes of the contours do not differ significantly, the Fisher matrix fails to predict the physical boundary $w_{a}+w_{0}<0$ which is required to explain the accelerated expansion in the dark-energy cosmological model. Therefore, the full posterior shifts towards the negative range in $w_{a}$. 


\subsection{Baryon acoustic oscillations}

The primordial density fluctuations in the early Universe leave an imprint on the dark matter and galaxy distributions, which can be still observed at much later times. Baryon acoustic oscillations (BAOs) cause an enhancement of the galaxy correlation function at a comoving distance of about $150 \mathrm{Mpc}$. This feature can be used as standard ruler, and therefore serves as a measure of the geometry of the Universe [39-41].

In an ideal case, to encompass all the information of a BAO survey, the full threedimensional power spectrum or correlation function should be used for constraining cosmology. The analysis of the long-wavelength power spectrum requires careful modeling, and can increase the constraining power on the cosmological parameters [42, 43]. Here, we choose a simplified ansatz and model a spectroscopic BAO survey following Refs. [44] and [1]: this assumes that the observations yield information on the angular and line-of-sight comoving distances. Accordingly, the observables to enter the likelihood are the logarithms of the comoving angular diameter distance $d_{\mathrm{A}}(z)=d_{\mathrm{L}}(z) /(1+z)^{2}$ and the Hubble parameter $H(z)$. The statistical error of the former is

$$
\sigma_{d_{\mathrm{A}}}(z)= \begin{cases}0.011 \sqrt{\frac{V_{0}}{V(z)}}\left(\frac{z_{\mathrm{m}}}{z}\right)^{0.5} & z<z_{\mathrm{m}} \\ 0.011 \sqrt{\frac{V_{0}}{V(z)}} & z>z_{\mathrm{m}}\end{cases}
$$

where the different cases refer to the non-linear evolution of matter for $z<z_{\mathrm{m}}=1.4$. $V(z)=d_{\mathrm{A}}^{2}(z) H^{-1}(z) \Omega_{\mathrm{sky}} \Delta z$ is the comoving volume for a redshift bin with width $\Delta z$, and observed solid angle $\Omega_{\text {sky }}$. The reference volume is $V_{0}=2.16 h^{-3}$ Gpc. The statistical error of the Hubble parameter is modeled as $\sigma_{H}=1.74 \sigma_{d_{\mathrm{A}}}$. We add in quadrature a systematic contribution of $\sigma_{\text {sys }}=0.01 \sqrt{0.5 / \Delta z}$ to both errors. Typically, BAO forecasts use some combination of $H(z)$ and $d_{A}(z)$ as parameters, and then linearly transform to the dark-energy parameters $w_{0}$ and $w_{a}$ [45]. Strictly speaking, it is the validity of this linear transformation we investigate here: by construction the likelihoods on $H(z)$ and $d_{A}(z)$ are Gaussian. Since we use a Fisher matrix formalism, the errors on $w_{0}$ and $w_{a}$ follow from a linear transformation and are therefore also Gaussian. However, the relation between $H(z)$ and $d_{A}(z)$ is truly non-linear and the Fisher matrix formulation can not encompass this behaviour.

As for supernovae, we consider the two cases of $w_{a}$ being a fixed and a free parameter in the analysis, respectively. The results for a stage-IV, SKA-like survey, shown in Fig. 2, are similar to the SNIa case: for a constant $w_{a}$, the likelihood function is only slightly bent, and the Fisher matrix is a reasonable approximation. When including a free $w_{a}$ however, the likelihood function becomes highly non-Gaussian, and its shape cannot be described any more by the curvature at the maximum only. This is an example where the variance and figure of merit from the Fisher matrix would suggest larger constraints than using the full likelihood. The reason for this is the relatively broad, low-curvature region around the maximum likelihood, which is captured by the Fisher matrix; the true likelihood function however is falling off quickly and bending away from the initial direction, excluding large regions covered by the Fisher ellipse. This behaviour becomes clearer when considering Figs. 3 and 4. The sample cloud pictured in Fig. 3 shows the three-dimensional distribution of the MCMC. The $w_{a}$ value of the sample points is colour-coded, transitioning from green to blue with $w_{a}$ going from positive to negative. The plot shows that there is a non-negligible correlation between the dark-energy parameters, namely positive $w_{0}$ and highly negative $w_{a}$ values, which is consistent with the condition $w_{0}+w_{a}<0$. Fig. 4 illustrates that some 

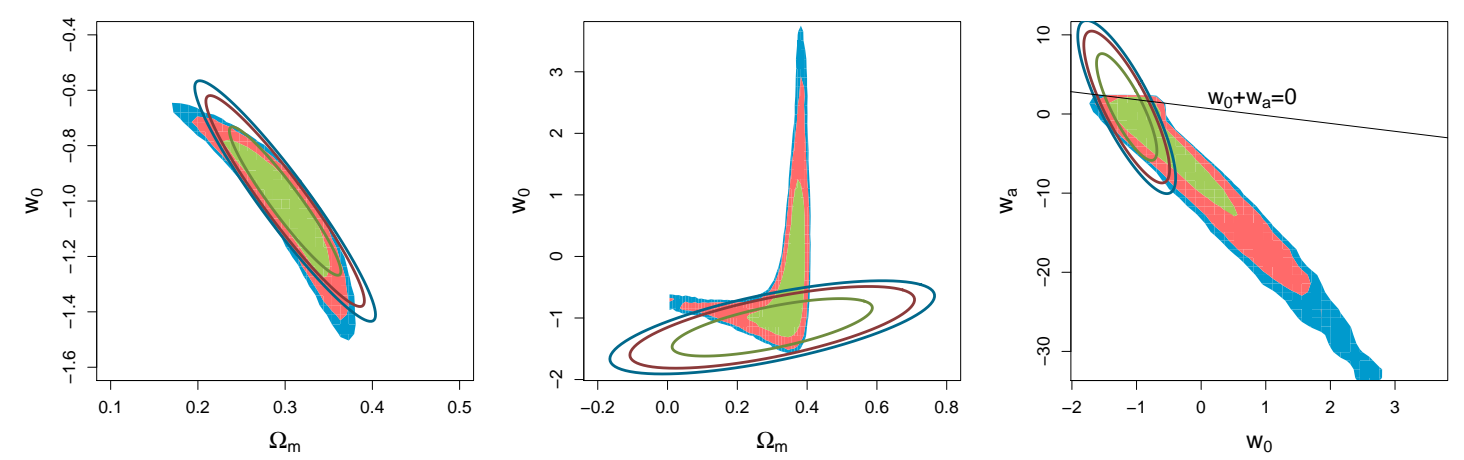

Figure 2. Confidence regions for a SKA-like BAO survey. Filled contours correspond to the MCMC, while the solid lines represent the Fisher matrix results. The parameter space is $\left\{\Omega_{\mathrm{m}}, w_{0}\right\}$ (left panel), and $\left\{\Omega_{\mathrm{m}}, w_{0}, w_{a}\right\}$ (middle and right panels). In the latter cases, we marginalise over the hidden parameter.

cosmological models with a highly non-standard expansion rate (red curve) generate angular diameter distances which are similar to the reference one with our LCDM fiducial parameters (black curve). This degeneracy of the CPL dark-energy parameters for geometrical probes originates from Eq. (3.3), where we can see that $w_{0}$ appears only in the sum with $w_{a}$. Since the only geometrical constraint is on the sum $w_{0}+w_{a}<0$, non-standard cosmological models cannot be ruled out with these data alone. The highly bent shape of the contours can be explained further by the green line in Fig. 4, which shows that the angular diameter distance for a cosmological model with $\Omega_{\mathrm{m}}=0.5$ is departing significantly from the reference model at redshifts $z>0.5$.

Although the total area of the confidence level contours is smaller for the MCMC forecasts, the variance of the dark-energy parameters is underestimated by the Fisher matrix also in this case. This introduces a bias on the DETF FoM of a factor of seven, as also shown in Table 2.

We show in Fig. 5 the forecasts for a HETDEX-like stage-III survey. As indicated in Table 1, as HETDEX is a high-redshift survey with relatively small sky coverage, it will not be able to put tight constraints on $w_{a}$, since in the CPL parameterisation dark energy is not a dominant component of the Universe at this epoch. In our forecasts we vary the parameters $\left\{\Omega_{\mathrm{m}}, w_{0}\right\}$ with flat priors $w_{0}>-3.0$ (left panel of Fig. 5) and $w_{0}>-10$ (right panel), respectively. These plots give further evidence that the Fisher matrix underestimates the variances of the dark-energy parameters. Since $w_{a}=$ const $=0$, the contours bend towards negative values of $w_{0}$, to a much larger extend than for SNIa (compare to the left panel of Fig. 2). The Fisher matrix is not able to predict the long tail of the posterior distribution, even for relatively moderate priors on $w_{0}$.

\subsection{Weak lensing}

Weak gravitational lensing is the distortion of images of distant galaxies caused by intervening matter along the line of sight between the source and the observer. Weak lensing is sensitive to both the geometry of the Universe, and the distribution of matter forming the large-scale structure. By measuring the weak-lensing distortions from galaxies as a function of their 


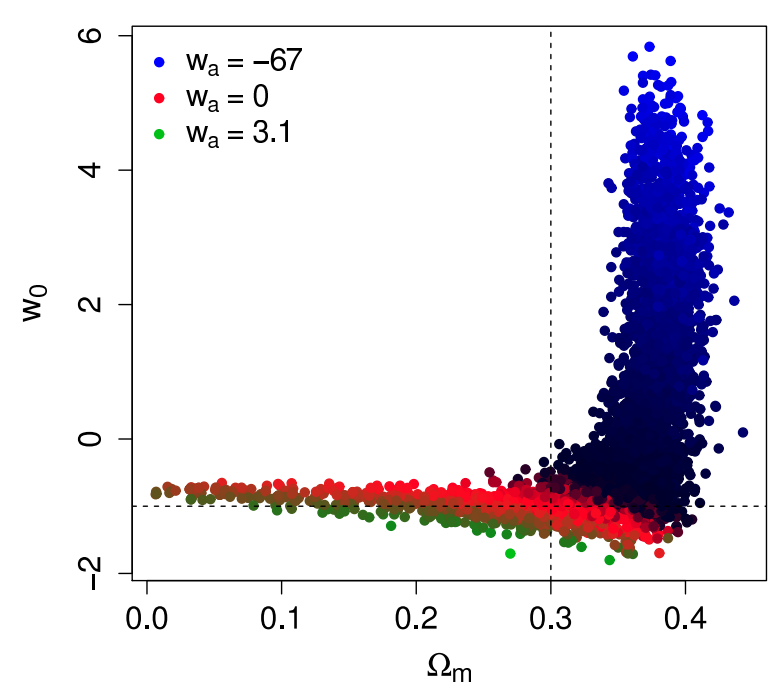

Figure 3. Plot of the accepted Markov chain sample points for a SKA-like BAO survey. The colour indicates the value of $w_{a}$, going from -67 (dark blue) to 3.1 (green).
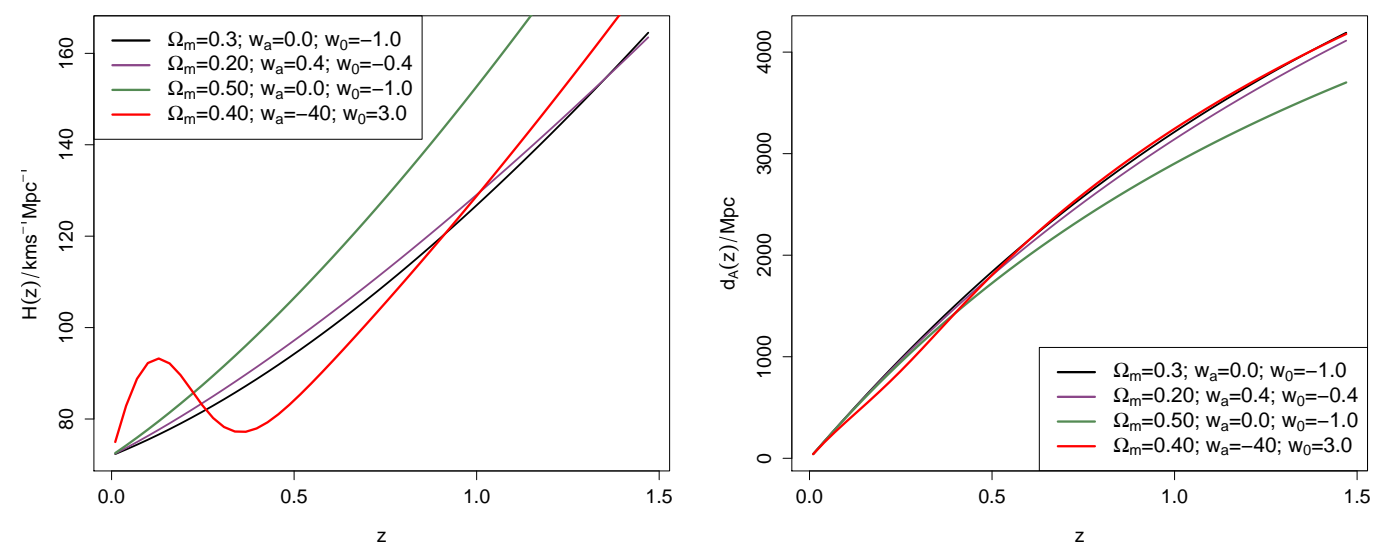

Figure 4. Expansion rate $H(z)$ (left) and comoving angular diameter distance $d_{\mathrm{A}}(z)$ (right) versus redshift for different cosmological models.

redshift (lensing tomography), information about the growth of structure can be deduced [46].

We model two large tomographic weak-lensing surveys with DES-like and Euclid-like settings, respectively. The $2 \mathrm{D}$ projected convergence power spectrum $C_{\ell}^{\kappa_{i} \kappa_{j}}$ between redshift bins $i$ and $j$ is given at any multipole $\ell$ by an integral over the three-dimensional matter power spectrum $P_{m}(z, k)[47,48]$,

$$
C_{\ell}^{\kappa_{i} \kappa_{j}}=\frac{9 H_{0}^{4} \Omega_{\mathrm{m}}^{2}}{4 c^{3}} \int \mathrm{d} z \frac{g_{i}(z) g_{j}(z)(1+z)^{2}}{H(z)} P_{m}\left(z, \frac{\ell}{\chi(z)}\right)
$$

where the wavenumber can be set to $k=\ell / \chi(z)$, given the comoving distance $\chi(z)$, by using the Limber approximation, which is accurate on all but the largest scales. The lens 

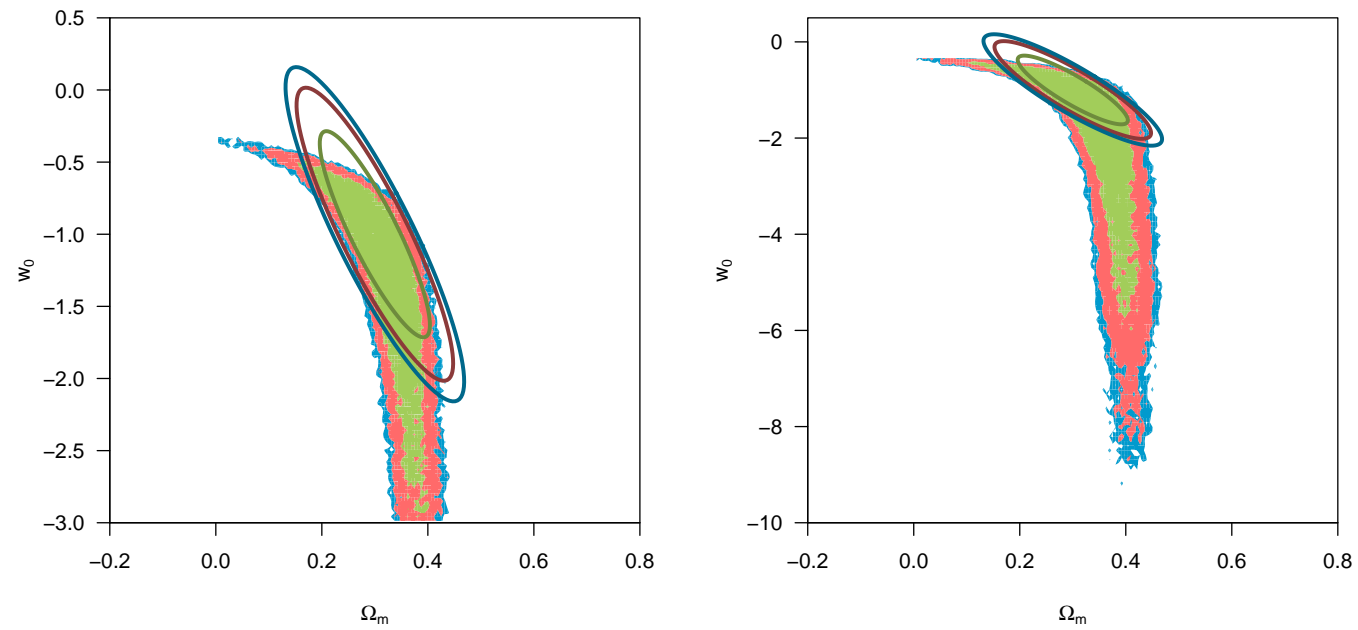

Figure 5. $\quad 68 \%, 90 \%$ and $95 \%$ confidence regions for a HETDEX-like BAO survey. The lower prior limit on $w_{0}$ is -3 (left panel) and -10 (right panel), respectively. No further free parameters are used. Filled contours correspond to the MCMC, while the solid lines represent the Fisher matrix results.

efficiency factor $g_{i}(z)$ is the equivalent of the geometrical ratio of the lens-source to observersource distances, weighted with the galaxy distribution of the corresponding redshift bin. For simplicity, we ignore photometric redshift errors, and we write the lens efficiency as an integral over the redshift bin width:

$$
g_{i}(z)=\int_{z_{i}}^{z_{i+1}} \mathrm{~d} z^{\prime}\left[1-\frac{\chi(z)}{\chi\left(z^{\prime}\right)}\right] \varphi\left(z^{\prime}\right) .
$$

Here $\varphi(z)$ represents the full galaxy redshift distribution, which is taken as [49]

$$
\varphi(z) \propto\left(\frac{z}{z_{0}}\right)^{\alpha} e^{-\left(\frac{z}{z_{0}}\right)^{\beta}} ; \quad \alpha=2, \beta=1.5, z_{0}=z_{\mathrm{med}} / 1.4
$$

where the median redshift $z_{\text {med }}$ is given in Table 1 for both survey settings. The redshift distribution is split into $N$ redshift bins between $z_{\min }$ and $z_{\max }$, such that the number of galaxies is the same in each bin (see Table 1 for the values corresponding to DES and Euclid).

We model the linear three-dimensional matter power spectrum with spectral index $n_{\mathrm{s}}=$ 0.96 and the transfer function from Ref. [50]. Baryonic damping is approximated by modifying $\Gamma \equiv \Omega_{\mathrm{m}} h$ according to Ref. [51].

We use the convergence power spectrum in the multipole range $0<\ell<1000$. On the smallest of these scales, the density power spectrum is already in the mildly non-linear regime. However, we choose the linear power spectrum for computational ease, and since the comparison of forecasting methods will not be affected as long as we use the same assumption for both cases. On intermediate scales, $100<\ell<1000$, where cosmic variance is already nonGaussian, and shot noise not yet dominating, the non-Gaussian contribution to the covariance is not negligible. The errors increase and mode-coupling between different multipoles is 

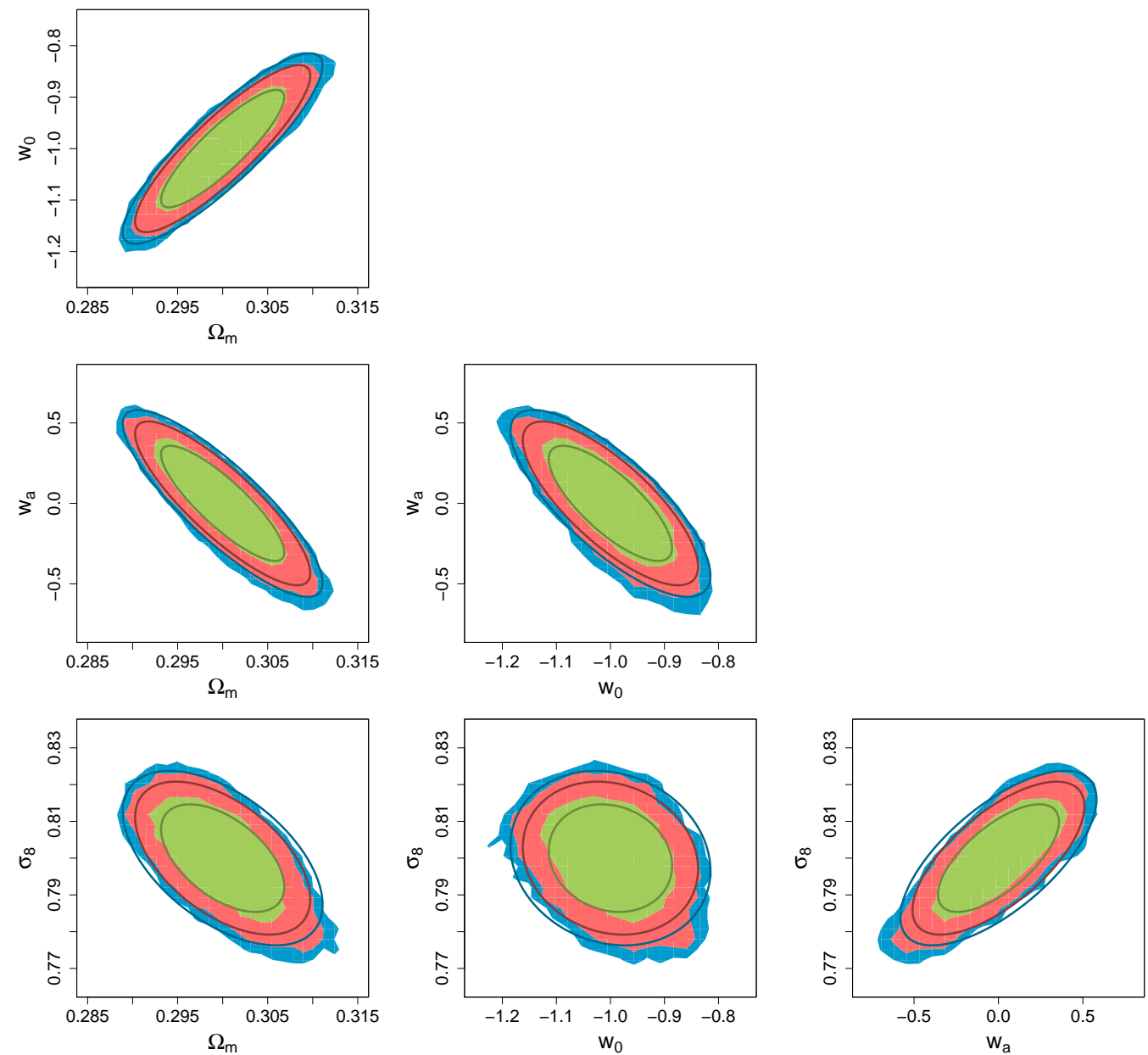

Figure 6. Marginalised 68\%, 90\% and 95\% confidence regions for a DES-like weak-lensing survey. Filled contours correspond to the MCMC, while the solid lines represent the Fisher matrix results.

introduced [52]. However, the resulting underestimation of cosmological parameters is only at the $10 \%$ to $20 \%$ level [53].

The comparison of the Fisher ellipses with full likelihood contours for a DES- and Euclidlike survey are shown in Figs. 6 and 7, respectively. The results of the method comparison for both surveys are similar, and the shapes and sizes of the confidence contours are in this case in agreement for the two methods, since the likelihood function features fairly elliptical contours for all parameter combinations. These results are in accordance with a previous analysis in [54].

\section{Towards Gaussian Parameters}

We have shown in the previous sections that the Fisher matrix technique can have severe weaknesses in predicting sizes and shapes of confidence level contours. The contours predicted by distance measure probes are not well-described by the Fisher matrix. The degeneracy of the dark-energy parameters $w_{0}$ and $w_{a}$ in the CPL parameterisation in the expansion rate 

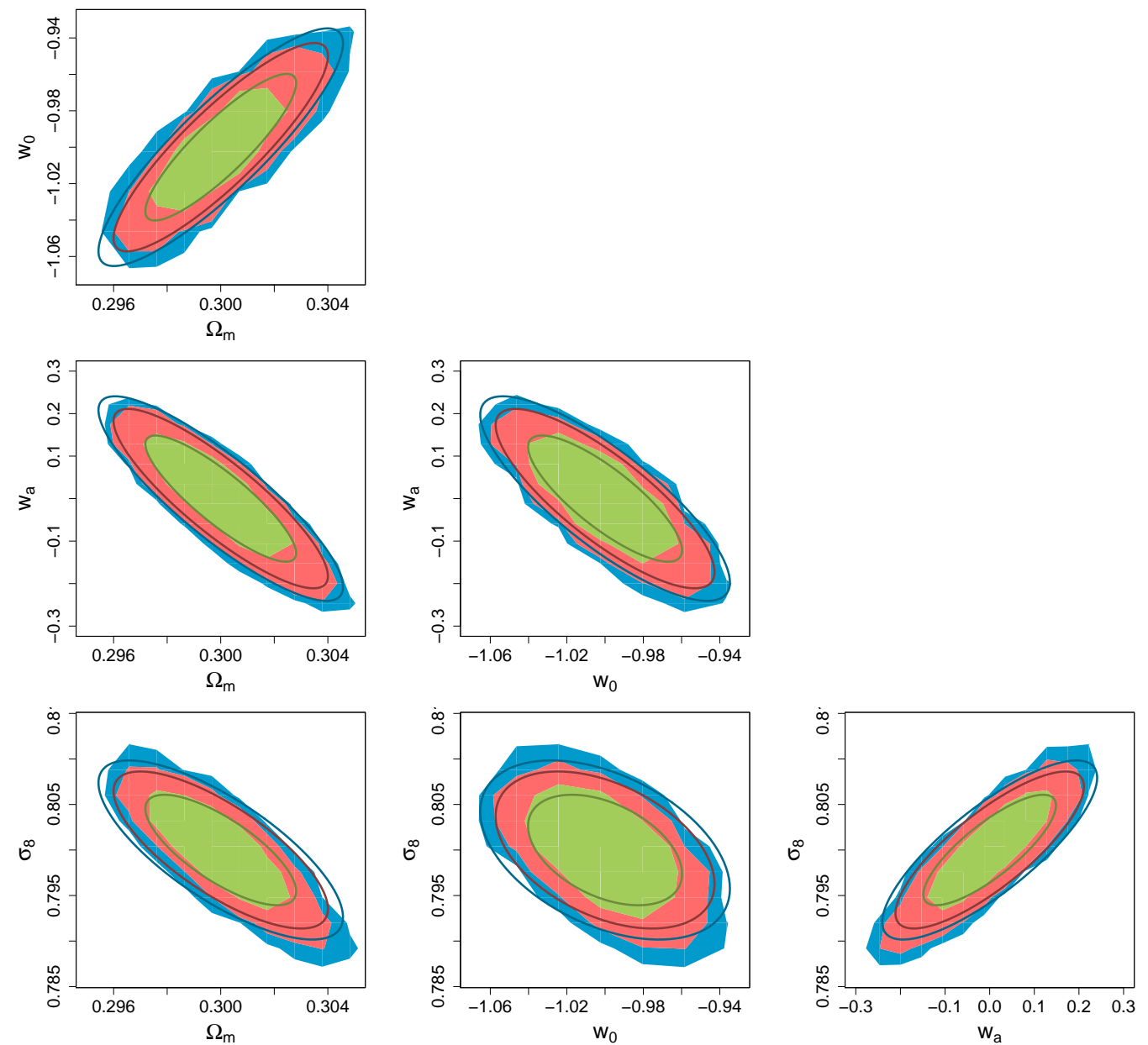

Figure 7. Marginalised 68\%, 90\% and 95\% confidence regions for a Euclid-like weak-lensing survey.

cannot be broken, and therefore a Gaussian approximation of the likelihood is not valid. For this reason, we introduce a new parameterisation, and suggest that SN and BAO Fisher matrix forecasts based on distance measures only should be considered in this new parameter space.

First, we define a new parameter $w_{s} \equiv \ln \left[-\left(w_{0}+w_{a}\right)\right]$ in order to exclude the region with $w_{0}+w_{a}>0$, which is clearly ruled out by the MCMC, as shown in Figs. 1 and 2. Motivated by the primary parameterisations of the BAOs and $\mathrm{CMB}$, we introduce the luminosity distance $d_{\mathrm{L}}\left(z_{\text {equ }}\right)$ as the basis of a new parameter. However, unlike in the case of CMB, there is no unique redshift for SN, at which the observables are measured. The only scale which is singled out in the redshift domain is the redshift of dark-matter and dark-energy equality $z_{\text {equ }}$, which is defined by

$$
\Omega_{\mathrm{m}}\left(1+z_{\text {equ }}\right)^{3}=\Omega_{\Lambda} e^{-3 w_{a} \frac{z}{1+z_{\text {equ }}}}\left(1+z_{\text {equ }}\right)^{3\left(1+w_{0}+w_{a}\right)} .
$$

We transform the MCM chain into the new parameter space $\left\{w_{s}, d_{\mathrm{L}}\left(z_{\text {equ }}\right)\right\}$ and perform a new Fisher matrix calculation. We restrict our analysis to the SN probe since SN and $\mathrm{BAO}$ show the same characteristics, and are based on the same analytical expressions. The 

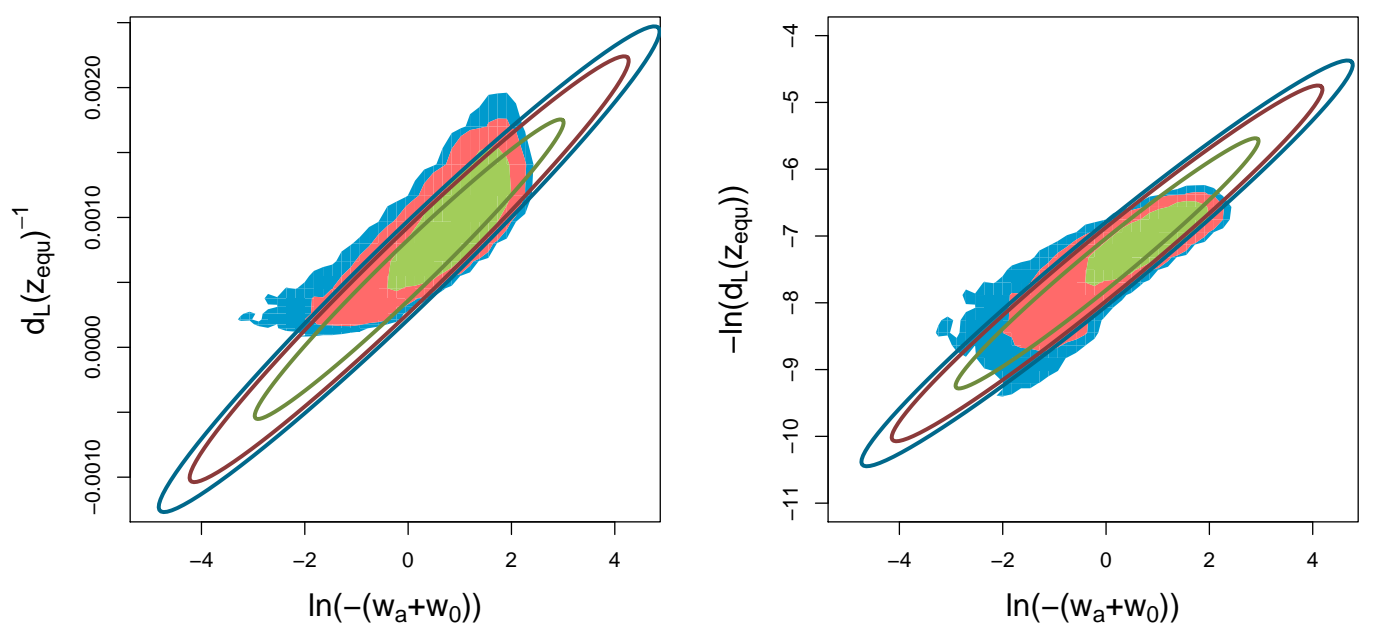

Figure 8. $68 \%, 90 \%$ and $95 \%$ confidence regions in new parameterisations, marginalised over $\Omega_{\mathrm{m}}$, for a supernova survey. Filled contours correspond to the MCMC, while the solid lines represent the Fisher matrix results.

\begin{tabular}{|l|c|c|c|c|}
\hline Survey & $\delta_{\Omega_{\mathrm{m}}}$ & $\delta_{w_{0}}$ & $\delta_{w_{a}}$ & FoM $_{\text {Fisher }} /$ FoM $_{\mathrm{MCMC}}$ \\
\hline SN & 1.43 & 0.40 & 0.86 & 1.20 \\
BAO SKA & 2.52 & 0.308 & 0.463 & 0.74 \\
BAO HETDEX & 0.62 & 0.38 & - & - \\
WL DES & 0.934 & 0.940 & 0.921 & 1.30 \\
WL Euclid & 1.01 & 1.04 & 0.968 & 1.12 \\
\hline
\end{tabular}

Table 2. For each of the three parameters $\Omega_{\mathrm{m}}, w_{0}$ and $w_{a}$, and for the five surveys settings, the values show the ratio of marginalised variances for the Fisher matrix over the MCMC, $\delta_{\Omega_{\mathrm{m}}}, \delta_{w_{0}}$ and $\delta_{w_{a}}$ respectively. A ratio smaller than unity means that the Fisher matrix underestimates the variance of the full posterior distribution. The fourth column shows the ratio of the DETF FoM, where we defined the FoM as the inverse of the area of the 1- $\sigma$ contour.

bare luminosity distance at $z_{\text {equ }}$ is still near-degenerate with $w_{s}$; therefore we apply another non-linear transformation by taking the inverse and the logarithm, respectively. Taking the logarithm ensures the positive definiteness of the luminosity distance. The result is shown in Fig. 8 for the new parameters $\boldsymbol{\theta}_{1}=\left\{w_{s}, d_{\mathrm{L}}^{-1}\left(z_{\text {equ }}\right)\right\}$ and $\boldsymbol{\theta}_{2}=\left\{w_{s},-\ln \left[d_{\mathrm{L}}\left(z_{\text {equ }}\right)\right]\right\}$, respectively.

The luminosity distance $d_{\mathrm{L}}\left(z_{\text {equ }}\right)$ depends via $z_{\text {equ }}$ on $\Omega_{\mathrm{m}}, w_{0}$ and $w_{a}$. However in the process of marginalisation over one parameter, in this case $\Omega_{\mathrm{m}}$, information is lost. This implies the irreversibility of the transformation and we have to compare models with the likelihood contours in this transformed parameter space. 


\section{Discussion and Conclusions}

We have investigated the comparison of the two forecast methods under the following four aspects: their practicality in application, the morphology of the marginalised confidence contours, the impact on the DETF FoM, and the Gaussianity of parameter combinations.

- Practicality: The advantage of the Fisher matrix forecast over MCMC is the computational speed. However, the Fisher matrix relies on the stability of numerical derivatives of the observables or the likelihood. To ensure high enough accuracy, it is necessary to probe many points corresponding to different step sizes $h$ for the numerical derivative (adaptive or non-adaptive). Furthermore, compared to the requirements for MCMC, a much higher numerical accuracy for each of the cosmological quantities is required. This is true in particular for parameters which have a subtle effect on the likelihood, such as $w_{a}$. As a consequence, the tabulation of recurrent quantities (such as the growth factor or the angular diameter distance) as well as the numerical integrations have to be undertaken with much higher precision. This will result in a increased computation time for one model by a factor of many. Fisher matrix forecasts will be even more demanding for more complex and more realistic calculations, for example, using non-linear models of the large-scale structure, full Boltzmann integrator for the transfer function, departures from the Limber approximation of the power spectrum, and correlations between cosmological probes [55].

- Morphology: On one hand, for purely geometrical probes such as supernovae, and more evidently BAOs, the likelihood function is not elliptical, and is asymmetric. Extended degeneracies exist between the parameters, since any combination thereof which reproduces the correct distance measure is allowed. However, other regions are excluded: for example firstly, if the sum $w_{0}+w_{a}$ is positive, then dark energy at late times scales faster than matter, which is disfavoured by the data. Secondly, the likelihood is zero for a negative matter density parameter $\Omega_{\mathrm{m}}$. The likelihood of the Fisher Gaussian approximation reaches well into these 'forbidden' regions, while being naturally unable to trace the extended degeneracies which in reality exist. The existence of such forbidden regions prevents us from defining parameter transformations which produce fully Gaussian likelihood distributions. An asymmetry due to physically forbidden regions always remains, making the Fisher matrix not a good approximation of the distribution.

On the other hand, for more complex observables which also trace structure formation, such as in our case weak lensing, all cosmological parameters enter the likelihood calculation in multiple ways: not only through the Hubble expansion, but also via the transfer and growth functions. This means that degeneracies are significantly alleviated, as we have shown in Figs. 6 and 7 . The same is likely to happen for a full tomographic galaxy clustering analysis, and especially so when additional features, such as redshift-space distortions and the Alcock-Paczynski effect, are included in the calculation $[10,55]$.

- Impact on the figure of merit: The ratio of the variances estimated from the Fisher matrix to the one corresponding to MCMC, as shown in Table 2, is expected to be smaller than unity in order to satisfy Eq. (2.4). With the exception of the ratios for $\Omega_{\mathrm{m}}$ for SN and BAO SKA (as discussed in Section 3.3), this is indeed the case. The largest mismatch using the Fisher matrix occurs for the purely geometrical probes, and 
in particular for the SKA BAOs. Interestingly the deviations in the Figure of Merit, which we define as the inverse of the area of the $68 \%$ likelihood contour, is not as large as the deviations on the single errorbars and morphologies of the contours. This is due to the different shape of the Gaussian contours, which often reach in an area, where the true likelihood is vanishing, and then underestimating the errors in other ranges. In addition, we should note that, the estimation of the area of the MCMC likelihoods has been performed on a relatively coarse grid, so it is also overestimated, this is in particular the case for the high accuracy WL surveys. Hence, with the exception of BAO SKA, we find the FoM in agreement on the $20 \%$ level with the Fisher matrix forecasts.

- Gaussianity: There have been previous studies on the Gaussianity of parameter combinations. For example, Ref. [18] lifted the degeneracy of parameters using the Box-Cox transformation [56]: they linearised the parameters in the $\Omega_{\mathrm{m}}-\sigma_{8}$ plane for a future weak-lensing survey. The approach worked well for the slightly curved contours in their case but, in the case of highly curved, non-Gaussian likelihood shapes such as for SN (see Fig. 1), we find that Box-Cox transformations do not provide a sufficient linearisation of the parameter to produce a Gaussian-like distribution.

Further, in Ref. [57] a comparison of forecasting methods to probe the large-scale structure is performed. They also found major differences of Fisher matrix and MCMC for small-volume surveys.

In this work, we have explored new, more Gaussian parameterisations, which are physically motivated. In this way, we succeed in linearising the parameter degeneracies, but the resulting likelihood function is still sufficiently non-Gaussian, that the Fisher matrix approximation remains relatively poor. In addition it is not possible to use the transformation we suggest to make the joint two-dimensional constraints on $w_{0}$ and $w_{a}$ more representative with the Fisher matrix approach. The reason for this is that after marginalisation over the other parameters, it is not possible to take the inverse of the transformation, since it also depends on $\Omega_{\mathrm{m}}$. Of course it would be possible to perform a numerical marginalisation (integration) in the full 3-dimensional backtransformed parameter constraints. But then the question arises how advantageous the Fisher matrix approach is in the first place, and whether one is not better off by performing a MCMC analysis. However, there might still a possibility that there is a useful parameterisation just involving $w_{0}$ and $w_{a}$.

We conclude from our comparisons that the Monte Carlo sampling analysis is preferable in the purely geometric cases, for which the Fisher matrix method has significant weaknesses in predicting parameter constraints regarding the morphology and orientation of the degeneracies.

When using simple probes of the expansion history, such as supernovae, the long parameter degeneracies are missed by the Fisher approach, while the problem is alleviated by the use of more complex tests of structure formation, such as weak lensing, for which we confirm previous results on the Gaussianity of the posterior probability. We highlight that we have not explicitly included CMB priors on the parameters, nor combined the probes with the WMAP (or Planck) likelihood. Although WMAP or Planck parameter constraints have no significance on the dark-energy parameters $w_{0}$ and $w_{a}$, they are able to break degeneracies with respect to $\Omega_{\mathrm{m}}$. Naturally, the combination of probes will tighten all constraints and 
make the likelihoods more Gaussian. However, in order to explore the merits of a single probe, we deem such a procedure misleading. Moreover, in general also the CMB likelihood deviates from Gaussianity and strong parameter degeneracies can be present [19].

In conclusion we advocate the full MCMC likelihood forecast method in order to explore realistically the merits of future dark energy surveys with geometrical probes of the expansion history; more generally the full MCMC method should be applied for any setting (combination of model parameters and observables), in which the Gaussianity of the likelihood distribution is unknown.

\section{Acknowledgments}

We would like to thank Sarah Bridle, Henk Hoekstra, Tom Kitching, Michael Kopp and Eric Linder for useful discussions. The work of MK, JW and TG is supported by the DFG TRR 33 'The Dark Universe'.

\section{References}

[1] A. Albrecht et. al., Report of the Dark Energy Task Force, astro-ph/0609591 (2006) [astro-ph/0609591].

[2] M. Chevallier and D. Polarski, Accelerating Universes with Scaling Dark Matter, International Journal of Modern Physics D 10 (2001) 213-223, [arXiv:gr-qc/0009008].

[3] E. V. Linder, Exploring the Expansion History of the Universe, Physical Review Letters 90 (2003), no. 9 091301, [arXiv:astro-ph/0208512].

[4] M. G. Kendall and A. Stuart, The Advanced Theory of Statistics, vol. II. London: Griffin, 1969.

[5] M. Tegmark, A. Taylor, and A. Heavens, Karhunen-Loève Eigenvalue Problems in Cosmology: How Should We Tackle Large Data Sets?, Ap. J. 480 (1997) 22, [arXiv:astro-ph/9707265].

[6] M. Tegmark, Measuring cosmological parameters with galaxy surveys, Phys. Rev. Lett. 79 (1997) 3806-3809.

[7] A. Rassat et. al., Deconstructing Baryon Acoustic Oscillations: A Comparison of Methods, arXiv e-prints (2008) [arXiv:0810.0003].

[8] A. Kosowsky, M. Milosavljevic, and R. Jimenez, Efficient cosmological parameter estimation from microwave background anisotropies, Phys. Rev. D 66 (2002), no. 6063007.

[9] A. Lewis and S. Bridle, Cosmological parameters from CMB and other data: A Monte Carlo approach, Phys. Rev. D 66 (2002), no. 10 103511-+.

[10] H.-J. Seo and D. J. Eisenstein, Probing Dark Energy with Baryonic Acoustic Oscillations from Future Large Galaxy Redshift Surveys, Ap. J. 598 (2003) 720-740, [arXiv:astro-ph/0307460].

[11] H. Hoekstra and B. Jain, Weak Gravitational Lensing and Its Cosmological Applications, Annual Review of Nuclear and Particle Science 58 (2008) 99-123, [arXiv:0805.0139].

[12] M. Bartelmann, TOPICAL REVIEW Gravitational lensing, Classical and Quantum Gravity 27 (2010), no. 23233001.

[13] S. W. Allen, A. E. Evrard, and A. B. Mantz, Cosmological Parameters from Observations of Galaxy Clusters, ARAEA 49 (2011) 409-470, [arXiv: 1103.4829].

[14] S. Perlmutter et. al., Measurements of the Cosmological Parameters Omega and Lambda from the First Seven Supernovae at $Z \geq 0.35$, Ap. J. 483 (1997) 565.

[15] A. Riess et. al., Observational Evidence from Supernovae for an Accelerating Universe and a Cosmological Constant, Astron. J 116 (1998) 1009. 
[16] R. Amanullah et. al., Light curves of five type Ia supernovae at intermediate redshift, A\&̈A 486 (2008) 375-382.

[17] D. H. Weinberg, M. J. Mortonson, D. J. Eisenstein, C. Hirata, A. G. Riess, and E. Rozo, Observational Probes of Cosmic Acceleration, submitted to Physics Reports (2012) [arXiv: 1201.2434].

[18] B. Joachimi and A. N. Taylor, Forecasts of non-Gaussian parameter spaces using Box-Cox transformations, MNRAS 416 (2011) 1010-1022.

[19] L. Perotto, J. Lesgourgues, S. Hannestad, H. Tu, and Y. Y Y Wong, Probing cosmological parameters with the CMB: forecasts from Monte Carlo simulations, JCAP 10 (Oct., 2006) 13, [astro-ph/].

[20] M. Martinelli, E. Calabrese, F. De Bernardis, A. Melchiorri, L. Pagano and R. Scaramella, "Constraining Modified Gravity with Euclid," Phys. Rev. D 83 (2011) 023012 [arXiv:1010.5755 [astro-ph.CO]].

[21] F. De Bernardis, M. Martinelli, A. Melchiorri, O. Mena and A. Cooray, "Future weak lensing constraints in a dark coupled universe," Phys. Rev. D 84 (2011) 023504 [arXiv:1104.0652 [astro-ph.CO]].

[22] N. Metropolis, A. W. Rosenbluth, M. N. Rosenbluth, A. H. Teller, and E. Teller, Equation of state calculations by fast computing machines, Journal of Chemical Physics 21 (1953) 1087-1092.

[23] W. Hastings, Monte carlo samping methods using markov chains and their applications, Biometrika (1970) 97-109.

[24] A. Gelman and D. B. Rubin, Inference from iterative simulation using multiple sequences, Statist. Sci. 7 (1992) 457-511.

[25] Schrabback et. al., Evidence of the accelerated expansion of the Universe from weak lensing tomography with COSMOS, A 6 A 516 (2010) A63+, [arXiv:0911.0053].

[26] F. Beutler et. al., The 6dF Galaxy Survey: baryon acoustic oscillations and the local Hubble constant, MNRAS 416 (2011) 3017-3032, [arXiv: 1106.3366].

[27] B. A. Reid et. al., Cosmological constraints from the clustering of the Sloan Digital Sky Survey DR7 luminous red galaxies, MNRAS 404 (2010) 60-85, [arXiv:0907.1659].

[28] S. Ho et. al., Clustering of Sloan Digital Sky Survey III Photometric Luminous Galaxies: The Measurement, Systematics and Cosmological Implications, arXiv e-prints (2012) [arXiv: 1201.2137].

[29] R. Kessler et. al., First-Year Sloan Digital Sky Survey-II Supernova Results: Hubble Diagram and Cosmological Parameters, Ap. J. Suppl. 185 (2009) 32-84, [arXiv:0908.4274].

[30] M. Sullivan et. al., SNLS3: Constraints on Dark Energy Combining the Supernova Legacy Survey Three-year Data with Other Probes, Ap. J. 737 (2011) 102, [arXiv: 1104.1444].

[31] N. Suzuki et. al., The Hubble Space Telescope Cluster Supernova Survey. V. Improving the Dark-energy Constraints above $z>1$ and Building an Early-type-hosted Supernova Sample, Ap. J. 746 (2012) 85, [arXiv: 1105.3470].

[32] E. Komatsu et. al., Seven-year Wilkinson Microwave Anisotropy Probe (WMAP) Observations: Cosmological Interpretation, Ap. J. Suppl. 192 (2011) 18, [arXiv:1001.4538].

[33] The Dark Energy Survey Collaboration, The Dark Energy Survey, arXiv e-prints (2005) [arXiv:astro-ph/0510346].

[34] G. Hill, K. Gebhardt, E. Komatsu, N. Drory, P. MacQueen, et. al., The Hobby-Eberly Telescope Dark Energy Experiment (HETDEX): Description and Early Pilot Survey Results, ASP Conf.Ser. 399 (2008) 115-118, [arXiv:0806.0183]. 
[35] R. Laureijs et. al., Euclid Definition Study Report, arXiv e-prints (2011) [arXiv:1110.3193].

[36] C. A. Blake, F. B. Abdalla, S. L. Bridle, and S. Rawlings, Cosmology with the Square Kilometre Array, New Astron.Rev. 48 (2004) 1063-1077.

[37] S. Rawlings, Cosmology with the Square Kilometre Array, arXiv e-prints (2011) [arXiv: 1105.6333].

[38] M. M. Phillips, The absolute magnitudes of Type IA supernovae, Ap. J. Letters 413 (1993) L105-L108.

[39] D. J. Eisenstein et. al., Detection of the Baryon Acoustic Peak in the Large-Scale Correlation Function of SDSS Luminous Red Galaxies, Ap. J. 633 (2005) 560-574, [arXiv: astro-ph/0501171].

[40] S. Cole et. al., The 2dF Galaxy Redshift Survey: power-spectrum analysis of the final data set and cosmological implications, MNRAS 362 (2005) 505-534, [arXiv:astro-ph/0501174].

[41] L. Anderson et. al., The clustering of galaxies in the SDSS-III Baryon Oscillation Spectroscopic Survey: Baryon Acoustic Oscillations in the Data Release 9 Spectroscopic Galaxy Sample, arXiv e-prints (2012) [arXiv: 1203.6594].

[42] A. G. Sánchez, C. M. Baugh, and R. Angulo, What is the best way to measure baryonic acoustic oscillations?, MNRAS 390 (2008) 1470-1490, [arXiv:0804.0233].

[43] F. Montesano, A. G. Sánchez, and S. Phleps, Cosmological implications from the full shape of the large-scale power spectrum of the SDSS DR7 luminous red galaxies, MNRAS 421 (2012) 2584, [arXiv:1107.4097].

[44] C. Blake, D. Parkinson, B. Bassett, K. Glazebrook, M. Kunz, and R. C. Nichol, Universal fitting formulae for baryon oscillation surveys, Mon.Not.Roy.Astron.Soc. 365 (2006) 255-264, [arXiv: astro-ph/0510239].

[45] H.-J. Seo and D. J. Eisenstein, Baryonic Acoustic Oscillations in Simulated Galaxy Redshift Surveys, Ap. J. 633 (2005) 575-588.

[46] M. Bartelmann and P. Schneider, Weak gravitational lensing, Physics Reports 340 (2001) 291.

[47] N. Kaiser, Weak gravitational lensing of distant galaxies, Ap. J. 388 (1992) 272-286.

[48] N. Kaiser, Weak Lensing and Cosmology, Ap. J. 498 (1998) 26-42.

[49] C. Baugh, S. Cole, C. Frenk, and C. G. Lacey, The Epoch of galaxy formation, Astrophys.J. 498 (1998) 504, [arXiv:astro-ph/9703111].

[50] J. M. Bardeen, J. R. Bond, N. Kaiser, and A. S. Szalay, The statistics of peaks of gaussian random fields, Ap. J. 304 (1986) 15-61.

[51] N. Sugiyama, Cosmic Background Anisotropies in Cold Dark Matter Cosmology, Ap. J. Suppl. 100 (1995) 281-+.

[52] J. Pielorz, J. Rödiger, I. Tereno, and P. Schneider, A fitting formula for the non-Gaussian contribution to the lensing power spectrum covariance, A\&A 514 (2010) A79, [arXiv: 0907.1524].

[53] M. Takada and B. Jain, The impact of non-Gaussian errors on weak lensing surveys, MNRAS 395 (2009) 2065-2086, [arXiv:0810.4170].

[54] A. N. Taylor and T. D. Kitching, Analytic Methods for Cosmological Likelihoods, accepted by MNRAS (2010) [arXiv:1003.1136].

[55] T. Giannantonio, C. Porciani, J. Carron, A. Amara, and A. Pillepich, Constraining primordial non-Gaussianity with future galaxy surveys, Mon.Not.Roy.Astron.Soc. 422 (2012) 2854-2877 [arXiv: 1109.0958]. 
[56] G. Box and D. Cox, An analysis of transformations, Journal of the Royal Statistical Society. Series B (Methodological) 26 (1964), no. 2 211-252.

[57] A. Hawken, F. Abdalla, G. Hutsi, and O. Lahav, Calibrating the BAO scale using the CMB: Lifting the degeneracy between geometric and dynamic distortions using the sound horizon from the CMB, submitted to MNRAS (2011) [arXiv:1111.2544]. 